\title{
Performance Benefits of Small Cells and Radio Optimization in Co-Channel Deployments
}

\author{
Mark C. Reed ${ }^{\ddagger \star}$ and He Wang ${ }^{\ddagger \star}$ \\ UNSW, Canberra ${ }^{\ddagger}$ \\ The Australian National University* \\ Email: mark.reed@unsw.edu.au
}

\begin{abstract}
Performance of outdoor small cells with co-channel deployment of macro cells for DS-CDMA systems is considered in this paper. Although interference management techniques, such as self organising networks, have been developed and to some degree deployed there are many open issues especially related to the performance of small cells. Small cells address outdoor hotspots and have different characteristics to indoor self-installed femtocells, including higher power levels and open subscriber group connectivity. This paper describes downlink performance results to better understand key parameters and trade-offs for these co-channel deployments. We describe the parameter configuration, the amount of offload achieved, and throughput for users connected to small cells. The results show that orders of magnitude of throughput improvements can be achieved when as little as four small cells per macro are deployed. We also discuss self optimisation use cases and show some motivating performance results.
\end{abstract}

\section{INTRODUCTION}

Mobile wireless communication systems today have benefited enormously from the segmented cellular architecture which has enabled enormous gains in throughput and performance. Mobile broadband demand, however, is forecast to increase rapidly in the coming years, where Cisco predicts a 18 times increase in the next five years [1]. The inherent interference limitations mean a solution is needed to minimise interference and maximise average throughput per unit volume. Small cells are a low cost, standard compliant, wireless access point using highly integrated chip technology originally designed for femtocells [2,3]. Small cells have significant differences from femtocells in that they are typically deployed outside in hotspot wireless areas to achieve a capacity dataoffload from the macro network. Small cells have a higher maximum transmit power (larger cell size) and also utilise open subscriber group mechanisms meaning that all users can join the small cell.

There is a growing body of research on two-tiered small cell networks, however not many results on open access small cells. System simulation results for small cells have been presented in [4-6] where system performance was measured. Coverage and capacity analysis of small cells, focusing on the downlink, has been undertaken in $[7,8]$. Reverse link capacity results for small cells and modeling results for inter-cell interference are provided in [9]. They modelled interference as a Gaussian random variable which is not always consistent with practical systems. Interference management has been the focus of several papers including $[10,11]$. In [12] the authors developed new analytical techniques to optimize small cell systems, and $[13,14]$ have considered interference coordination.

Although small cell networks are currently being rolled out in many parts of the world, comparatively little research has been undertaken to model and optimize these systems [3]. Two-tiered small cell networks re-use spectrum (i.e. reuse $=$ 1) enabling massive increases in the bits $/ \mathrm{sec} / \mathrm{Hz} / \mathrm{m}^{3}$. Recent work by the Small Cell Forum considers a similar system model as we address in this paper [15], where we provide unique differences to this work with fixed number of small cell locations, a different distribution of the users: where we hard limit to a maximum number of users for a cell sector, different shadowing, and different macro antenna array models. These differences are more practical where the constraint on locations of small cells is due to city streets and the number of small cells per sector is limited by deployment costs. This spectrum re-use creates significant interference, while more random-like cell locations changes the network layout from the theoretical grid layout. Co-existence of small and large-scale cells introduces a new network layout which we study here where high transmit powers in the macro-cell can completely swamp the signal in a nearby small cell. We also consider Self optimising networks (SON) which are being actively developed and deployed by wireless vendors [16, 17], discussing use cases and summarise criteria.

In this paper we exclusively study open access small cells to determine downlink performance for a WCDMA/HSPA system. We determine the number of user equipment (UE) terminals that are served by the macro and small cell when a different number of small cells per macro are deployed. We consider the downlink throughput improvement due to small cell deployments. We highlight the process in determining these results through simulation where multiple experiments (drops) are conducted to provide statistically averaged results. We also consider Self Optimising use cases and highlight some results for these criteria.

\section{SySTEM MODEL}

In this work, we consider the downlink scenario of a two-tier heterogeneous cellular network, where conventional macrocell and co-channel small cells are deployed in the outdoor environment, which is the focus of the interference analysis of the small cells. The small cells operate in open access mode by providing service to all the users that meet the 
cell association requirements where the strongest pilot channel signal $(\mathrm{CPICH})$ will determine the terminals association. Part of the macro and small cell layout is shown in Figure 1 where the macro cellular structure has 3 sectors and 7 base stations (total of 21 sectors) with a base station spacing of 500 meters. There are ten potential small cell locations spaced as shown in the figure. In our model the possible small cell locations are pre-selected (as shown) and are randomly utilized depending on how many small cells are deployed in the macro cell. Unlike most work which uses random distributed cell sites we use fixed locations as we believe this to be more realistic due physical restrictions of the street grid in large cities which limits location of small cells (typically attached to the side of buildings).

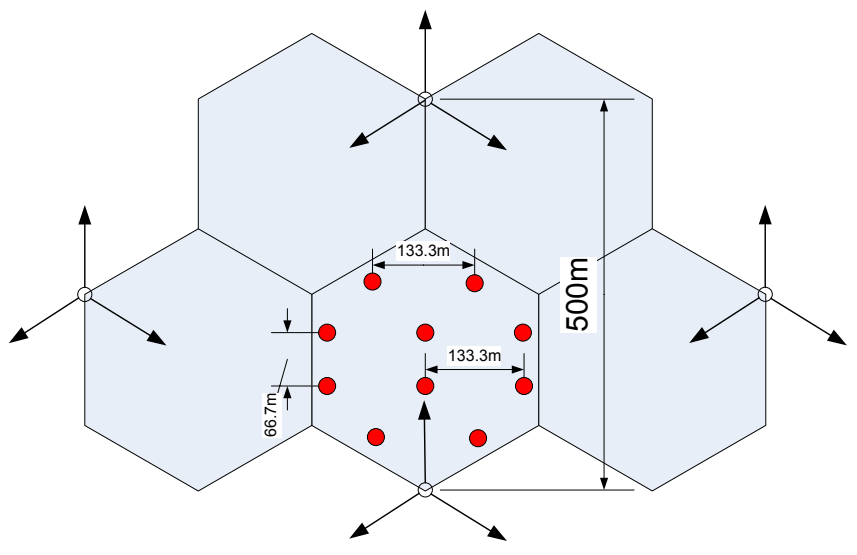

Fig. 1: Macro and Small Cell Layout

The Radio Frequency (RF) propagation and simulation models in this paper are listed in the Table I. The parameters are typical values used where the macro base station maximum power is $43 \mathrm{dBm}$ and the small cell maximum power is $30 \mathrm{dBm}$ with a $0 \mathrm{dBi}$ omni-directional antenna gain. The terminals assume Type-3 which includes Linear Minimum Mean Square Equaliser (LMMSE) chip equalisation and two receiver antennas.

The channel model parameters are detailed in Table II where the path loss, probability of Line of Sight (LOS), log normal shadowing parameters, terminal speed (walking speed), and fading model (Single path Rayleigh) are listed. Because of the outdoor environment, the path loss model used here follows the one in [18], which decide whether or not a link is LOS, determined from the distance between the transmitter and the receiver.

The number of experiments (drops) conducted with different user locations, where 10,000 transmission time intervals (TTIs)are simulated for each drop. As there are correlated shadowing models used it is important to simulate a lot of TTIs for any given experiment.

\begin{tabular}{|c|c|}
\hline Operating Spectrum & $2100 \mathrm{MHz}$ \\
\hline $\begin{array}{l}\text { Number of Shared Macro Carriers } \\
\text { Available for Femtocells }\end{array}$ & Single Carrier (Co-channel) \\
\hline Number of Macrocell Sites & 7 \\
\hline Number of Sectors per Site & 3 \\
\hline Site-to-Site Spacing & $500 \mathrm{~m}$ \\
\hline Max. Macro NodeB Tx Power & $43 \mathrm{dBm}$ \\
\hline Macrocell Antenna Model & $\begin{array}{c}G(\theta)=G_{\max }-\min \left[12\left(\frac{\theta}{\beta}\right)^{2}, G_{s}\right],-\pi \leq \theta \\
\leq \pi\end{array}$ \\
\hline \multirow[t]{3}{*}{ Macrocell Antenna Parameters } & $\begin{array}{l}\text { Angle where gain pattern } \\
\text { is } 12 \mathrm{~dB} \text { down from peak }\end{array}$ \\
\hline & Sidelobe gain level in $\mathrm{dB}$ \\
\hline & Max. gain level in $\mathrm{dB}$ \\
\hline Max. Small Cell NodeB Tx Power & $30 \mathrm{dBm}$ \\
\hline Small Cell NodeB Tx Power Algorithm & Fixed \\
\hline Femto NodeB Antenna & Omni, $0 \mathrm{dBi}$ \\
\hline Number of SC Candidate Locations & 10 \\
\hline Number of Small Cells in one Sector & $\begin{array}{l}0,1,4,10 \text { (randomly chosen } \\
\text { from candidate locations) }\end{array}$ \\
\hline CPICH Power Ratio (Macro and SC) & $10 \%$ \\
\hline HS-DSCH Power Ratio (Macro and SC) & $40 \%$ \\
\hline No. of Spreading Codes for HS-DSCH & 15 \\
\hline Scheduling Algorithm & Proportional Fairness \\
\hline UE Antenna Model & Omni, OdB \\
\hline UE Category & Cat-10 \\
\hline UE Type & $\begin{array}{c}\text { Type-3 (LMMSE chip equalization and } 2 \mathrm{Rx} \\
\text { antenna) }\end{array}$ \\
\hline UE Receiver Noise Figure & $13 \mathrm{~dB}$ \\
\hline No. of UEs in one Sector & 30 \\
\hline
\end{tabular}

TABLE I: System Parameter for Macrocells and Small Cells.

\begin{tabular}{|c|c|}
\hline $\begin{array}{c}\text { Path Loss Model } \\
\text { (Macro to UE) }\end{array}$ & $\begin{array}{c}\mathrm{PL}_{\operatorname{Los}}(\mathrm{R})=123.4+24.2 \log 10(\mathrm{R}) \\
\mathrm{PL}_{\mathrm{NLO}}(\mathrm{R})=151.1+42.8 \log 10(\mathrm{R})\end{array}$ \\
\hline Probability of LOS (Macro to UE) & $\operatorname{Prob}(\mathrm{R})=$ \\
& $\min (0.018 / \mathrm{R}, 1)^{*}(1-\exp (-\mathrm{R} / 0.063))+\exp (-\mathrm{R} / 0.063)$ \\
\hline $\begin{array}{c}\text { Path Loss Model } \\
\text { (SC to UE) }\end{array}$ & $\mathrm{PL}_{\operatorname{Los}(\mathrm{R})=103.8+20.9 \log 10(\mathrm{R})}$ \\
\hline PLNos $(\mathrm{R})=145.4+37.5 \log 10(\mathrm{R})$
\end{tabular}

TABLE II: Channel Model Parameters.

\section{System Simulation ApProach: Macrocell NETWORK OFFLOADING (DOWNLINK)}

The system simulation process consists of a number of steps as listed in Figure 2. Firstly the small cell locations are selected randomly from the provided locations and the terminals are dropped with a uniform distribution in the sector. The pathloss, shadowing and pilot channel power is determined for each terminal before the long term high speed downlink shared channel Signal to Interference plus noise ratio (SINR) is 
calculated. For each transmission time interval (TTI) the SINR mapping for the LMMSE chip level equaliser is performed before the mapping from SINR to single terminal throughput. We assume that full buffer downlink data traffic is required for all 30 Type- 3 users where we use a proportional fair scheduler at both macro cell and small cell.

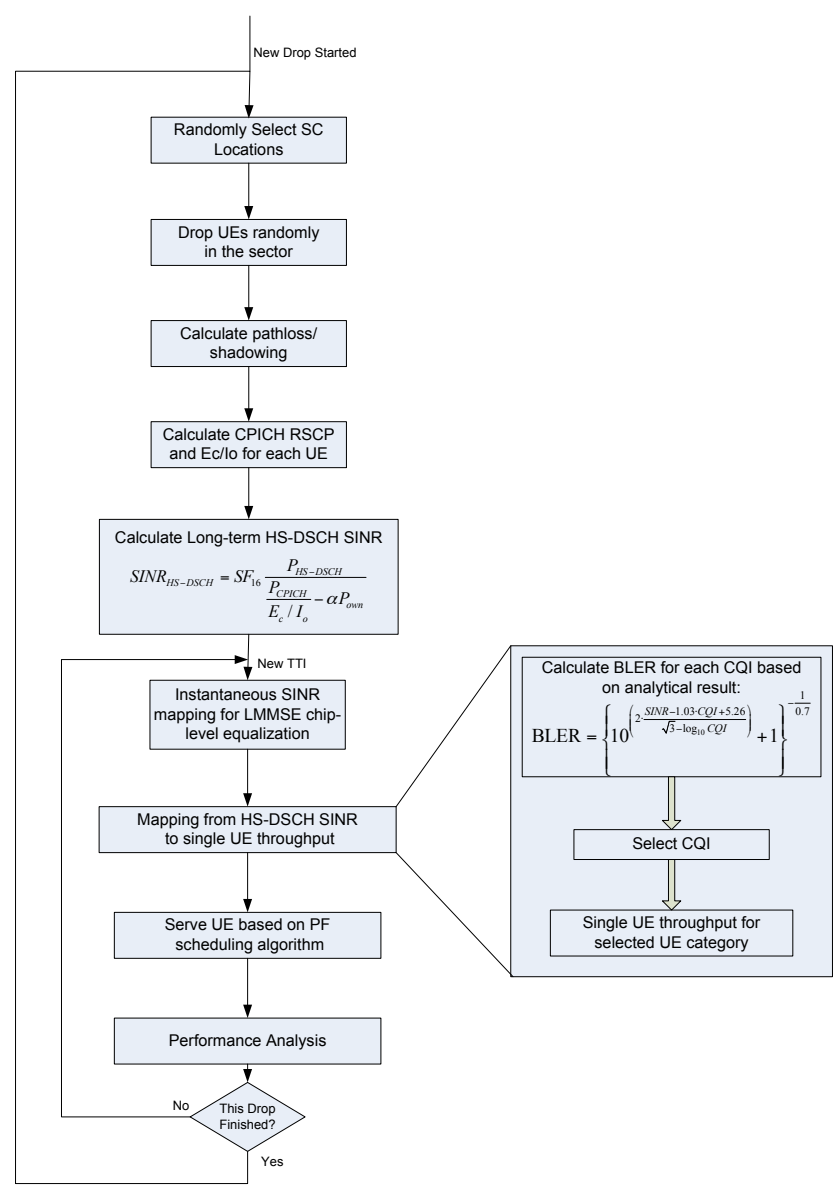

Fig. 2: Flow diagram of Simulation process

\section{Radio Optimisation With SElF Optimising NETWORKS (SON)}

Self optimising networks are being actively researched and developed by wireless vendors $[16,17]$. The key reasons for their deployment is minimising costs and managing the increasing complexity of the networks. Small cells are a large contributing factor to the complexity of the network and this means that SON is no longer just a benefit, it is essential for small cells and Heterogenous Networks (HetNets). Networks with small cell deployments mean that users are much more likely to be on the edge of cells and in handover regions, also increasing the user handover management. The sheer number of small cells that are expected to be deployed means that semi-manual radio parameter tweaking that currently is performed for large macro base stations does not efficiently scale to tens of thousands of cells.
Standardisation work on SON has defined so-called use cases $[19,20]$ to define the objectives of the optimisation and segment this into different objectives.

The key SON functions (or use cases) are:-

- PCI - Physical Cell ID Optimisation

- ANR- Automatic Neighbour Relations

- CCO- Coverage and Capacity Optimisation

- MDT - Minimising Drive Tests

- MLB - Mobility Load Balancing

- MRO - Mobiility Robutstness (handover) Optimisation

We briefly discuss two of these use-cases below. The objective of coverage optimisation is to maximise signal coverage by modifying downlink transmit power, and antenna tilt. The parameters can be modified from measurement campaigns or by users reporting signal strength. Compared to coverage optimisation the objective of capacity optimisation is to maximise the sum throughput across the users and their individual throughput. The techniques for performing optimisation are generally proprietary and many approaches are utilised. The objectives of automatic neighbour relations (ANR) is to automatically determine the neighbouring cells which is necessary information needed by the infrastructure for efficient handover management where this can improve mobility robustness. The nearest neighbours are not always those located physically close as shadowing and fading can severely distort the cell boundaries leading to unpredictable neighbour relations.

There are a number of ongoing challenges for SON, for example each of these use-case algorithms do not operate in isolation, instead the use-case algorithms interact in conflicting ways where an increase in one parameter can cause another algorithm to decrease the same parameter potentially causing the use-case algorithms to fight each other, leading to poor performance outcomes for the network. Solutions to this problem include ordering the SON use-cases and developing algorithms that jointly optimise for particular parameters. A further challenge for SON is the Macro and small cell interactions. The sharing of parameters for ANR and Handover optimisation (MRO) becomes necessary in a HetNet, thus a mechanism for parameter sharing is needed. Another significant challenge with SON is multi-vendor interoperation. Each set of vendor equipment needs mechanisms to communicate to other vendors equipment. The approach will be different for centralised and distributed SON.

Finally future SON will more than likely consist of interradio access technology (Inter-RAT) where optimisation is performed between $3 \mathrm{G}, 4 \mathrm{G}$ and WiFi. Being able to pick the best performing technology for any given user would clearly be a benefit to overall network.

\section{NUMERICAL RESULTS}

The key challenges in small cell deployments for wireless operators are determining

- The throughput improvement that small cell connected users would experience

- The amount of data offload (relief) from the macro cell (which can improve macro user throughput), and 
- The amount of uplink noise rise that small cell connected users generate onto the macro cell/s.

\section{A. Throughput Improvement with Small Cells}

In this section we consider the number of terminals offloaded and the throughput improvements that result, addressing the first two points. We leave the third point as future work. In this section we assume there are 30 active users per macrocell sector area, uniformly distributed. Figure 3 shows the $\mathrm{CDF}$ of the number of terminals served by the macro cell for differing number of small cells in the macro cell sector. When the number of small cells (SC) rises to 10 per macro sector then only at most nine users are served by the macro with at most 21 users are served by the small cells. This means that for a relatively small number of small cells a significant amount of data offload can result.

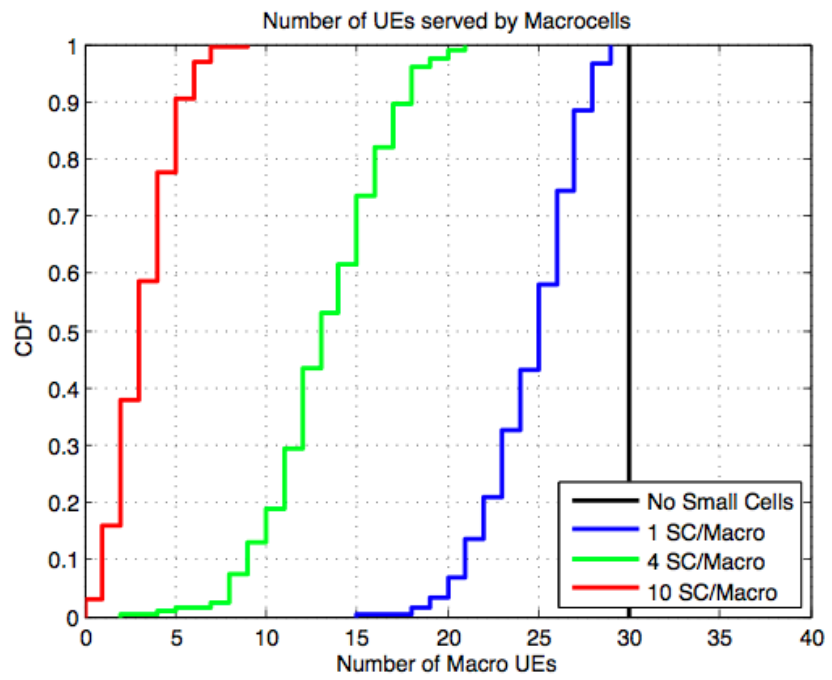

Fig. 3: Cumulative Distribution Function for Number of Terminals (UE) served by the Macrocell

In Figure 4 the cumulative distribution function of the number of terminals served by the small cells is shown. This is essentially the complementary result from Fig. 3. For 10 small cells per macro sector with 30 UEs per macro sector a minimum of 21 users are served by the small cells. As can be seen with a larger density of small cells more of the UEs are served by the small cells. As expected, the increase of the small cell density gives more chance to offload macrocell users to small cells.

In Figure 5 the cumulative distribution function of an individual user's throughput is shown (averaging macro and small cell connected users). It indicates that almost all the users, i.e. macro users and small cell users, benefit from the offloading from the macrocell to the small cells. This shows that for $70 \%$ of the users with ten small cells the throughput increases from less than $100 \mathrm{kbps}$ to more than $2 \mathrm{Mbps}$, an increase of 20 times. As more macro users are offloaded to the small cells, there are few macro users left to share the

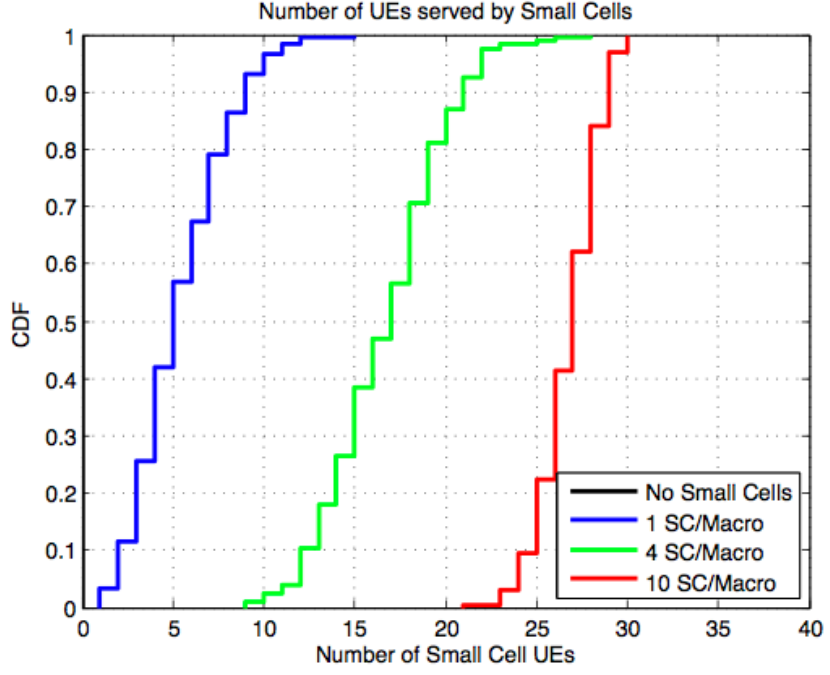

Fig. 4: Cumulative Distribution Function for Number of Terminals (UEs) served by Small Cells

resource of macro cell and significant improvement occurs for both the macro users and the small cell users.

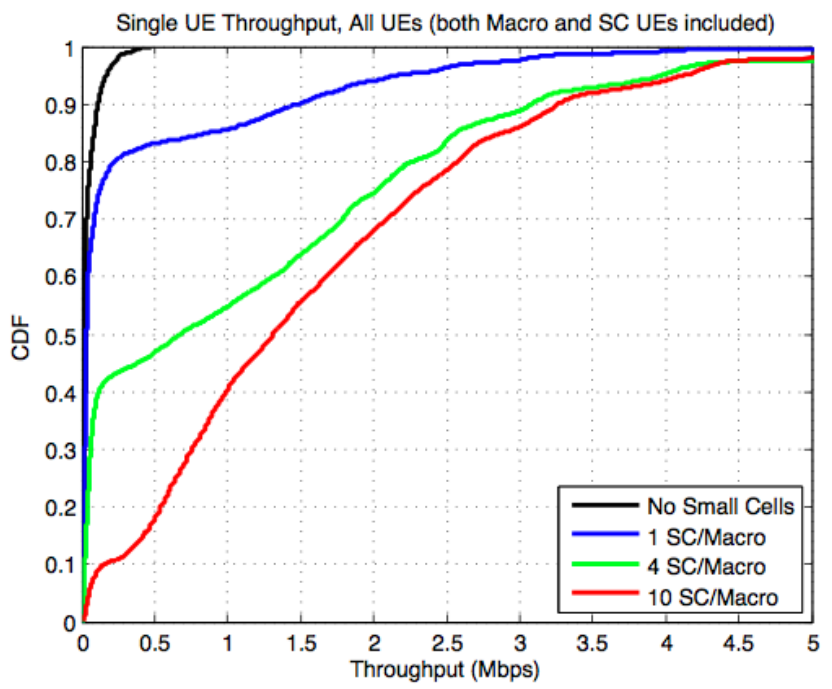

Fig. 5: Single User Throughput, averaged for both Macro and Small Cell connected users

Figure 6 shows the throughput improvement for a single macro terminal for different numbers of small cells. Although not as dramatic as the improvement for the small cell terminals the macro terminal still doubles it's data rate for $70 \%$ of the users, highlighting the improvement to macro users.

Finally we show the downlink throughput for a small cell connected terminal in Figure 7 for different numbers of small cells per macro sector. Increasing the density of small cells will make the interference worse in the region, which explains the slightly worse performance of the ten small cells per 


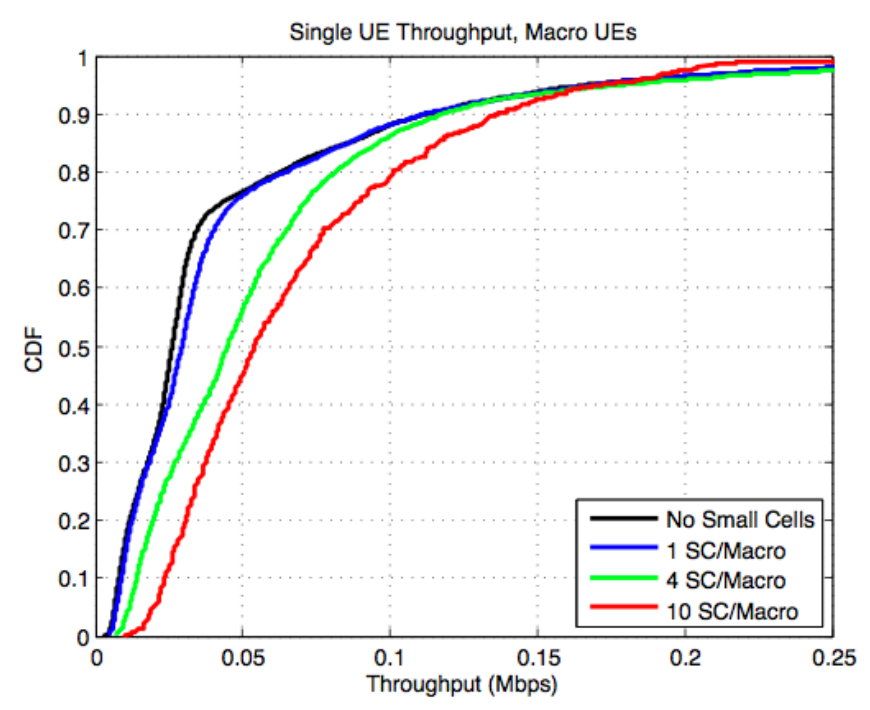

Fig. 6: Single UE Throughput for Small Cell UEs

macro (10 SC/macro) performance curve compared to the four SC/Macro. What should be noted is that the individual throughput is orders of magnitude greater than for the macro. Also noteworthy is that the sum throughput is still improved significantly since more users are served by small cells.

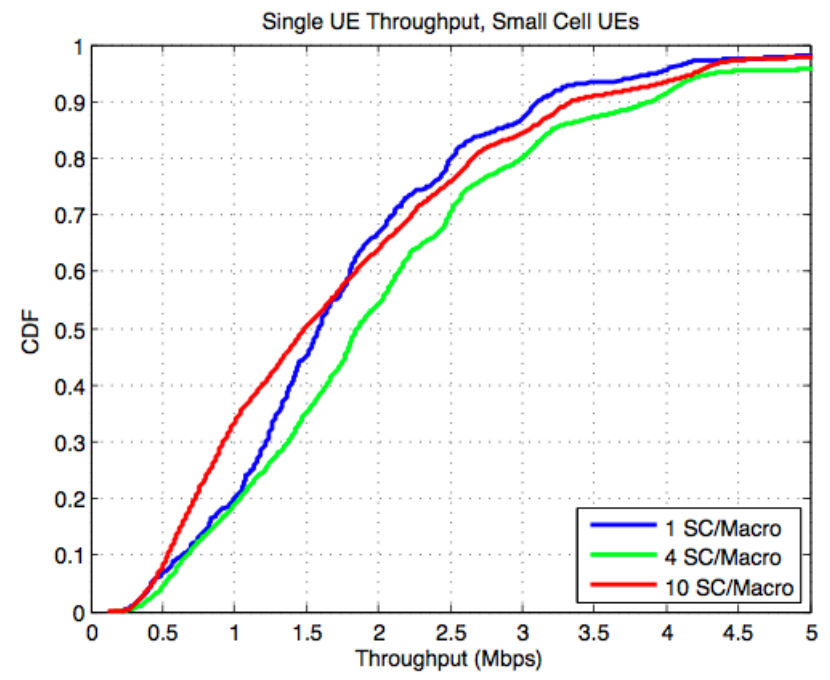

Fig. 7: Cumulative Distribution Function for Common Pilot Channel (CPICH)

\section{B. Self Organising Networks with Small Cells}

In this section we show some example optimisation results, we start with the outdoor environment and show signal strength heat maps and automatic neighbour relations (ANR) as two example results. In Fig 8 we show a signal strength heat map (red=strong signal, blue= weak signal) collected from a mobile network, this data has been used for visualisation as well as coverage and capacity optimisation. This data, collected from user terminals, highlights how radio network performance can be determined without expensive drive tests, thus being able to minimise drive test (MDT).

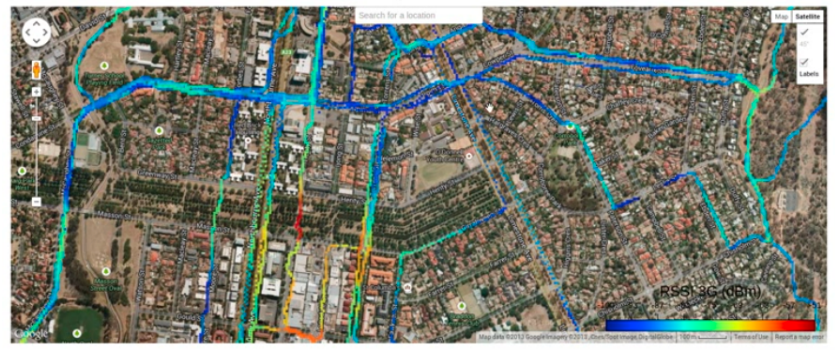

Fig. 8: Signal strength Heatmap determined from handset signal strength measurements

Automatically determining neighbouring base stations is important for wireless operators to efficiently and seamlessly enable users to handover to the next cell. Figure 9 shows a number of base stations as circles on the map. The base station of interest is marked in black and the green base stations have been determined to be neighbours. In this case the cell marked in red is not a neighbour. This illustrates that automatic neighbour relations (ANR) can be automatically determined and utilised for improving mobility (handover) robustness (MRO).

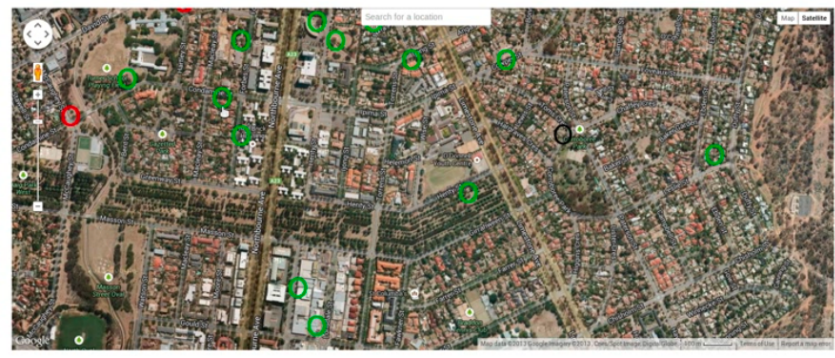

Fig. 9: Diagram showing Base station Neighbours (in green) for the Black Cell, Red Cell is not a neighbour

We also want to highlight coverage optimisation and to do this we utilise an indoor enterprise example. Based on the three levels of the LTE Reference Signal Received Quality (RSRQ) we use thresholds of -3 to $-11 \mathrm{~dB}$ as excellent (Red), -11 to $-13 \mathrm{~dB}$ as acceptable (Green), and -13 to $-19 \mathrm{~dB}$ as being poor (Blue). Figure 10 shows the power heat map for an enterprise building prior to optimisation where the small cell power levels are too high causing significant power leakage. This is not wanted as users can then connect outdoors and create significant uplink noise rise onto the outdoor network.

Figure 11 shows post-optimised power for this same enterprise building. Here it can be clearly seen that the power levels of the small cells in the enterprise have been reduced where indoor coverage is still acceptable and outdoor leakage has significantly reduced.

We have shown examples of Self Organisation for HetNets and highlighted the use cases as determined by 3GPP. We have 


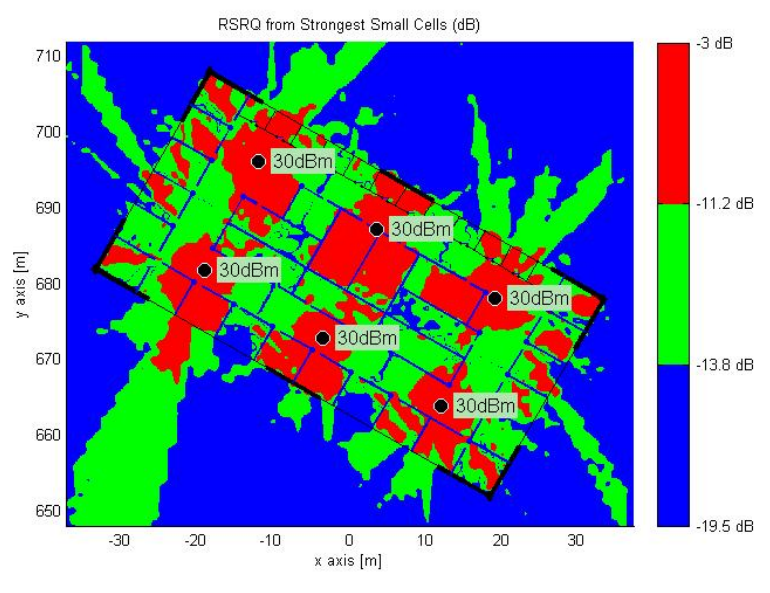

Fig. 10: Enterprise Building prior to radio optimisation of the RSRQ Level

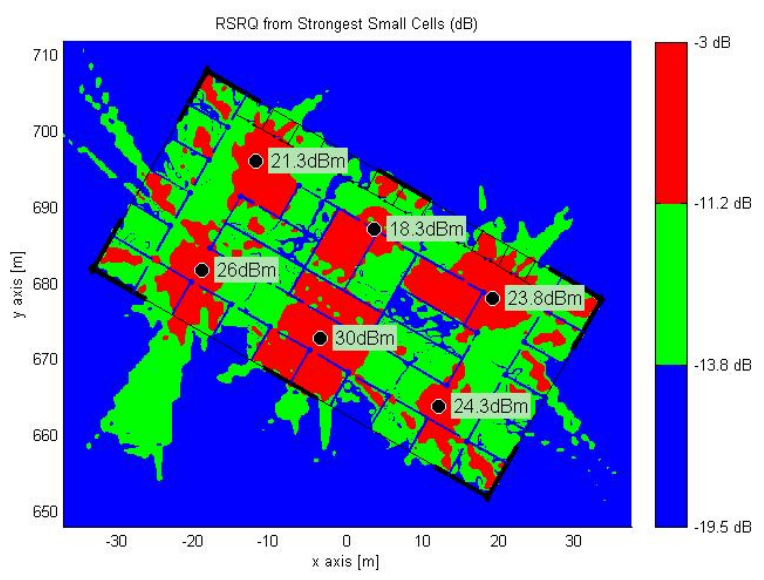

Fig. 11: Enterprise Building following radio optimisation of the RSRQ Level

demonstrated how SON can be used for determining neighbour relations, mobility robustness, and for coverage optimisation.

\section{SUMmary}

In this paper we have investigated the downlink performance of a $3 \mathrm{G}$ system where a 3-sectored macro cellular system has co-channel deployed small cells. We also discuss self optimising networks, including the motivation for them and the key use cases. We uniquely limited the number of users per macro cell sector and fix the the locations of the small cells. We determined the amount of users that can be offloaded for varying numbers of small cells, where the small cells are open access with higher power than for those used in typical femtocell deployments. The results have highlighted that with only a low number of small cells more than two thirds of the users would associate with a small cell and thus be offloaded from the system. Throughput improvements of up to 20 times are possible where realistic channel conditions and proportional fair scheduling is used for both the femtos and macros. The results highlight that macrocell users will also benefit by doubling their throughput. Finally, examples of radio optimisation are shown to highlight the potential benefits of automatic self optimisation networks to small cell networks.

\section{ACKNOWLEDGEMENT}

M. C. Reed was with UNSW, Canberra and affiliated with the Australian National University. He Wang was with the UNSW Canberra. This work was supported by the Australian Research Councils Discovery Projects funding scheme DP130101760.

\section{REFERENCES}

[1] "Cisco visual networking index : Global mobile data traffic forecast update 2011 - 2016," Feb. 2012.

[2] V. Chandrasekhar, J. Andrews, and A. Gatherer, "Femtocell networks: a survey," IEEE Commun. Mag., vol. 46, pp. 59-67, Sept. 2008.

[3] J. Andrews, H. Clausen, M. Doher, S. Rangan, and M. Reed, "Femtocells: Past, present and future," IEEE J. Selected Areas Commun., Apr. 2012.

[4] H. Claussen, "Co-channel operation of macro- and femtocells in a hierarchical cell structure," Int'l. J. Wireless Inform. Networks, vol. 15, no. 3-4, pp. 137-147, 2008.

[5] H. Claussen, L. Ho, and L. Samuel, "Self-optimization of coverage for femtocell deployments," in Proc. Wireless Telecommunications Symp. (WTS'08), (Pomona, USA), pp. 278-285, Apr. 2008.

[6] H. Claussen and F. Pivit, "Femtocell coverage optimization using switched multi-element antennas," in Proc. IEEE Int'l Conf. on Commun. (ICC'09), (Dresden, Germany), pp. 1-6, June 2009.

[7] V. Chandrasekhar, M. Kountouris, and J. Andrews, "Coverage in tiered cellular networks with spatial diversity," in Proc. IEEE Global Commun. Conf. (GLOBECOM'09), (Honolulu, USA), pp. 1-6, Dec. 2009.

[8] V. Chandrasekhar, M. Kountouris, and J. Andrews, "Coverage in multiantenna two-tier networks," IEEE Trans. Wireless Commun., vol. 8, pp. 5314-5327, Oct. 2009.

[9] V. Ramaswamy and D. Das, "Multi-carrier macrocell femtocell deployment-a reverse link capacity analysis," in Proc. IEEE 70th Vehic. Tech. Conf. (VTC'09-Fall), (Anchorage, USA), pp. 1-6, Sept. 2009.

[10] A. Schroder, H. Lundqvist, G. Nunzi, and M. Brunner, "User-assisted coverage and interference optimization for broadband femtocells," in Proc. 11th IFIP/IEEE Int'l Symp. on Integrated Network ManagementWorkshops (IM'09), (New York, USA), pp. 199-204, June 2009.

[11] M. Yavuz, F. Meshkati, S. Nanda, A. Pokhariyal, N. Johnson, B. Raghothaman, and A. Richardson, "Interference management and performance analysis of UMTS/HSPA+ femtocells," IEEE Commun. Mag., vol. 47, pp. 102-109, Sept. 2009.

[12] H. Wang, M. Zhao, and M. C. Reed, "Outage analysis for WCDMA femtocell with uplink attenuation," in IEEE Globecom 2010 Workshop on Femtocell Networks (FEMNet 2010), (Miami, U.S.A), Dec. 2010.

[13] L. Garcia, K. Pedersen, and P. Mogensen, "Autonomous component carrier selection: interference management in local area environments for LTE-advanced," IEEE Commun. Mag., vol. 47, pp. 110-116, Sept. 2009.

[14] D. Lopez-Perez, A. Valcarce, G. de la Roche, and J. Zhang, "OFDMA femtocells: a roadmap on interference avoidance," IEEE Commun. Mag., vol. 47, pp. 41-48, Sept. 2009.

[15] "W-CDMA open access small cells," Small Cell Forum, 2012.

[16] A. Fehske, I. Viering, J. Voigt, C. Sartori, S. Redana, and G. Fettweis, "Small-cell self-organizing wireless networks," Proceedings of the IEEE, vol. 102, pp. 334-350, March 2014.

[17] O. Aliu, A. Imran, M. Imran, and B. Evans, "A survey of self organisation in future cellular networks," Communications Surveys Tutorials, IEEE, vol. 15, pp. 336-361, First 2013.

[18] "3GPP TR 36.814 v9.0.0 E-UTRA: Further advancements for E-UTRA physical layer aspects."

[19] 3GPP, "Telecommunication management; self-organizing networks (SON); concepts and requirements," 3GPP TS 32.500, June 2010.

[20] NGMN, "Recommendation on SON and O\&M requirements," NGMN Requirement Specification, Dec. 2008. 